Disintegrin and metalloproteinases (ADAMs) expression in gastroesophageal reflux disease and in esophageal adenocarcinoma

Kauttu, T.

2017-01

Kauttu , T , Mustonen , H , Vainionpää , S, Krogerus , L , Ilonen , I , Rasanen , J , Salo , J \& Puolakkainen, P 2017 , ' Disintegrin and metalloproteinases (ADAMs) expression in gastroesophageal reflux disease and in esophageal adenocarcinoma ' , Clinical \& translational oncology , vol. 19 , no. 1 , pp. 58-66 . https://doi.org/10.1007/s12094-016-1503-3

http://hdl.handle.net/10138/232553

https://doi.org/10.1007/s12094-016-1503-3

publishedVersion

Downloaded from Helda, University of Helsinki institutional repository.

This is an electronic reprint of the original article.

This reprint may differ from the original in pagination and typographic detail.

Please cite the original version. 


\title{
Disintegrin and metalloproteinases (ADAMs) expression in gastroesophageal reflux disease and in esophageal adenocarcinoma
}

\author{
T. Kauttu ${ }^{1,2} \cdot$ H. Mustonen ${ }^{2,3}$ - S. Vainionpää ${ }^{3}$ L. Krogerus ${ }^{4}$ I. Ilonen ${ }^{1,2}$ \\ J. Räsänen ${ }^{1,2} \cdot$ J. Salo ${ }^{1,2} \cdot$ P. Puolakkainen ${ }^{2,3}$
}

Received: 19 October 2015/Accepted: 15 March 2016/Published online: 30 March 2016

(c) Federación de Sociedades Españolas de Oncología (FESEO) 2016

\begin{abstract}
Background Clinically useful marker molecules for the progression of gastroesophageal reflux disease and Barrett's esophagus (BE) to esophageal adenocarcinoma (EAC) are lacking. Many adenocarcinomas and inflammatory conditions exhibit increased expression of ADAMs, 'a disintegrin and metalloproteinases'.

Methods We assessed the expression of five ADAMs (9, $10,12,17,19)$ in three esophageal cell lines (Het-1A, OE19, OE33) by RT-PCR and Western blotting, and in human samples of normal esophagus, esophagitis, BE, Barrett's dysplasia, and EAC by RT-PCR, and in selected samples by immunohistochemistry.

Results EAC patients showed increased mRNA expression of ADAMs 9, 12, 17 and 19, as compared to controls. At immunohistochemistry, ADAM9 and ADAM10 proteins were increased in EAC. Patient samples also showed increased mRNA expression of ADAM12 in esophagitis, of
\end{abstract}

Electronic supplementary material The online version of this article (doi:10.1007/s12094-016-1503-3) contains supplementary material, which is available to authorized users.

P. Puolakkainen

pauli.puolakkainen@hus.fi; pauli.puolakkainen@helsinki.fi

1 Department of General Thoracic and Esophageal Surgery, Heart and Lung Centre, Helsinki University Hospital, Helsinki, Finland

2 Department of Surgery, Clinicum, Faculty of Medicine, University of Helsinki, Helsinki, Finland

3 Department of Gastroenterologic and General Surgery, Helsinki University Hospital, Haartmaninkatu 4, PO Box 340, 00290 Helsinki, Finland

4 Department of Pathology, HUSLAB, Helsinki University Hospital, Helsinki, Finland
ADAM9 in BE, and of ADAMs 9, 12 and 19 in Barrett's dysplasia, as compared to controls. Two EAC cell lines showed increased ADAM9 mRNA.

Conclusions ADAM9 expression is increased in EAC. Its predecessors show increased ADAM9 mRNA expression. The importance of the alterations in ADAM expression for the development of EAC, and their use as marker molecules, warrant further studies.

Keywords Esophageal adenocarcinoma . Gastroesophageal reflux disease · Barrett's esophagus . ADAM molecules

\section{Introduction}

Esophageal adenocarcinoma (EAC) is associated with a high overall mortality [1], in spite of the advances in its treatment. The prevention of EAC by treating its recognised predisposing factor, gastroesophageal reflux disease (GERD), or its sequel, Barrett's esophagus (BE), by surgery or medication has not proven effective [2-4]. Thus, the search for clinically useful markers of progression from $\mathrm{BE}$ to malignancy has been extensive but unsuccessful [5].

As there is no evidence to date of a single decisive transformation pathway leading to EAC, its development is likely to be multifactorial. A molecule subfamily of the metalloproteinases, a disintegrin and metalloproteinases (ADAMs), has been previously linked to both inflammation [6] and malignancy [7], and, therefore, was chosen to be studied in the inflammatory, metaplastic, dysplastic and carcinoma phases of GERD. ADAMs participate in the shedding of growth factors and in cellular adhesion [8]. Their composition entails them the capacity for multiple functions: they consist of eight domains, including a 
metalloproteinase and a disintegrin domain, a transmembrane sequence, and a prodomain, the removal of which activates the metalloproteinase domain [9]. Increased expression of ADAMs has been found in many cancers [7], and has been also linked to cancer progression and aggressiveness [10]. ADAMs are naturally inhibited by the tissue inhibitors of metalloproteinases (TIMPs) [7], and oncologic medication targeting them has been developed [10]. Increased ADAM expression has also been found in inflammatory bowel disease and Helicobacter pylori gastritis $[11,12]$. Multiple substrates of ADAMs, including transforming growth factor beta (TGF- $\beta$ ) and E-cadherin, have been shown to have a prognostic impact in EAC, although with no major individual clinical relevance [13]. ADAM mRNA expression has been found to be increased in esophago-gastric adenocarcinomas [14]. While defining the origin of the tumours in gastro-esophageal junction is difficult, it is important because of the probably differing etiology. For gastric adenocarcinoma, Helicobacter pylori infection is a strong risk factor, whereas EAC and its risk factor BE bear an inverse relationship with this infection [15].

We, thus, wanted to explore the importance of ADAMs in the GERD-related esophageal and gastroesophageal junctional (Siewert 1 and 2) cancers during their development process. The expression of five ADAM molecules (ADAMs 9, 10, 12, 17 and 19) was assessed at mRNA level, and partly at the protein level. Their expression at different stages of GERD was compared to that in the distal esophagus of normal controls, as well as to that in the proximal esophagus of the GERD and EAC patients. The expression of ADAMs was also analysed in cell lines of an esophageal and a gastroesophageal junctional adenocarcinoma, and from the normal esophagus. ADAM9 expression was increased in EAC at both the protein and mRNA levels, and ADAM9 mRNA was increased in BE and Barrett's dysplasia.

\section{Materials and methods}

The study protocol was approved by the Ethics Committee of the Department of Surgery, Helsinki University Hospital.

\section{Cell lines and cell culture}

Cell lines of Barrett's adenocarcinoma (OE33), and of adenocarcinoma of the gastroesophageal junction (OE19), the authenticity of which has been confirmed [16], were used. For comparison, Het-1A, a cell line drawn from normal esophageal tissue transfected with a SV40 large T antigen, was used. OE33 and OE19 were purchased from Health Protection Agency Culture Collections (Salisbury,
UK), and Het-1A and the gastric carcinoma cell line NCIN87 that served for standardization from LGC Promochem (Middlesex, UK). The cell lines were cultured in $37^{\circ} \mathrm{C}$ in media as follows: OE33, OE19 and NCI-N87 in RPMI $+10 \%$ FBS (fetal bovine serum) (Gibco, Carlsbad, CA), and Het-1A in BEGM ${ }^{\mathrm{TM}}$ Bronchial Epithelial Cell Growth Medium (Cambrex Bio Science, East Rutherford, NJ).

\section{Patient characteristics}

Samples were randomly collected from patients undergoing upper gastrointestinal endoscopy or esophageal surgery during years 2007-2010, and classified according to the histology (Table 1). Controls were patients with normal esophageal mucosa undergoing endoscopy for suspected upper gastrointestinal pathology. One patient was included in two different study groups, as first biopsied BE with mild dysplasia, and transformed later to EAC during follow-up.

\section{Tissue samples}

All patients gave a written, informed consent of their participation. Tissue samples were collected in upper gastrointestinal endoscopies, or at surgery due to esophageal adenocarcinoma or high-grade dysplasia. Two to three samples from three different locations were drawn for each patient from both the distal (approx. $2 \mathrm{~cm}$ above the gastroesophageal junction), and the proximal esophagus (at approx. $15 \mathrm{~cm}$ above the gastroesophageal junction). One sample was placed in RNAlater (Qiagen, Hilden, Germany) for RT-PCR, and other samples from the same location were fast-frozen in liquid nitrogen, or directly placed in $10 \%$ formalin for immunohistochemistry.

\section{Extraction of RNA and proteins}

RNA from the cell lines was isolated using the RNeasy Mini Kit (Qiagen, Germantown, MD) according to the protocol specified in the Kit. From the tissue samples, RNA was extracted by the RNeasy Fibrous Tissue Mini Kit from (Qiagen). Tissues were homogenized in the Thermo Savant FastPrep (Qbiogene, Carsbad, CA). RNA was then isolated according to the Kit protocol, except for the double amount of Buffer RLD and Proteinase K used in tissue homogenization. For all mRNA samples, the DNAse Kit (Qiagen, Hilden, Germany) was used to eliminate DNA.

Proteins from the cell lines were extracted using a buffer containing $150 \mathrm{mmol} \mathrm{NaCl}, 10 \mathrm{mmol}$ Tris-HCl, Tris-Base, 1 mmol EDTA, 1 mmol EGTA. $1 \%$ TritonX-100 and $0.5 \%$ $\mathrm{Np}-40$ with $\mathrm{pH}$ of 7.6. Proteinase inhibitors Roche's Complete block A ( $1 \mathrm{tbl} / 50 \mathrm{ml}$ ) and PMSF (phenylmethylsulfonyl fluoride) were added to the buffer before use. 


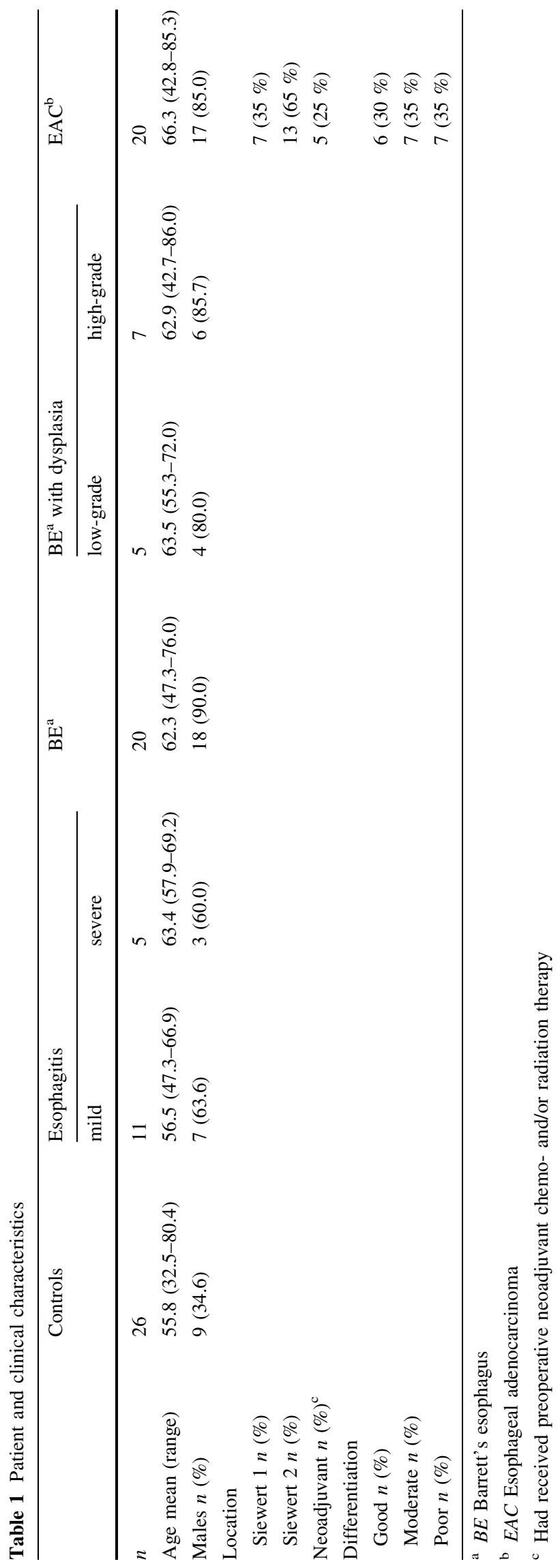

\section{Reverse transcription (RT)-PCR}

RNA was reversely transcripted to cDNA using the High Capacity cDNA Archive Kit (Applied Biosystems, Foster City, CA). RT-PCR was performed, and the amount of cDNA was quantified, with ABI Prism 7000 Sequence Detection Software exploiting Taqman technology. Primers were purchased from Applied Biosystems for ADAM9 (Hs00177638_m1), ADAM10 (Hs00153853_m1), ADAM12 (Hs01106104_m1), ADAM17 (Hs00234224_ $\mathrm{m} 1$ ), and ADAM19 (Hs00224960_m1). For the cell lines, RT-PCR for each ADAM was run in triplicate, and for the tissue samples, each was run in duplicate.

We used two methods for standardization of the RTPCR results: The same dilution batch of the cell line NCIN87 cDNA was run in every plate to count for plate to plate variations. The expression of glyceraldehyde-3-phosphate dehydrogenase (GAPDH) in cell lines, along with that of beta-actin (ACTB) in the tissue samples, was measured for each specimen and used for correction of the ADAM expression.

\section{Western blotting}

To quantify protein content of cell lines, we used the Quant-it kit (Invitrogen, Eugene, OR) with fluorescence plate reader (Wallac 1420, Perkin-Elmer, Turku, Finland). Extracted proteins were separated by SDS-electrophoresis on $10 \%$ polyacrylamide gel, and transferred to a nitrocellulose membrane (Bio-Rad, Hercules, CA). Odyssey blocking buffer (Li-Cor, Lincoln, NE) was used for blocking and antibody dilutions. To detect ADAMs, the membrane was incubated overnight with primary antibodies (Online Resource 1) in dilutions $1.0 \mu \mathrm{g} / \mathrm{ml}$. After incubating with fluorescent-labelled secondary antibodies (dilution 1:15 000) (Online Resource 1), the signal was measured with Odyssey infrared scanner (Li-Cor) and analysed with Odyssey software (v2.1). The GAPDH expression served for standardisation.

\section{Immunohistochemistry}

For immunohistochemistry, 6 controls, 3 patients with severe esophagitis, 3 with non-dysplastic BE, 5 with dysplastic BE, and 7 with esophageal adenocarcinoma were selected as the most representative samples, irrespective of their mRNA expression. Immunohistochemistry was performed on formalin-fixed, paraffin-embedded tissue samples. Antigen retrieval was performed by heating the glasses in $0.01 \mathrm{M} \mathrm{Na-citrate} \mathrm{buffer,} \mathrm{at} \mathrm{pH} 6$ and temperature of $98{ }^{\circ} \mathrm{C}$ for $20 \mathrm{~min}$. For blocking, Normal Horse Serum $2.5 \%$ (ImmPRESS ${ }^{\mathrm{TM}}$ Reagent Kit Universal AntiMouse/rabbit Ig, MP-7500, Vector Laboratories, 
Burlingame, CA) was used. Glasses were then incubated with primary antibodies overnight at $4{ }^{\circ} \mathrm{C}$. Antibodies were targeting ADAM9 (AB19024, dilution 1:400, Millipore (Billerica, MA)), ADAM10 (AB936, 1:750, R\&D Systems, Minneapolis, MN), ADAM12 (LS-B2756, 1:2000, LifeSpanBioSciences (Seattle, WA)), ADAM17, HPA010738100 UL, 1:50, Sigma-Aldrich (St.Louis, MO)), and ADAM19 (sc25988, 1:200, Santa Cruz Biotechnology, Dallas, TX). After that, slides were soaked with $0.75 \%$ $\mathrm{H}_{2} \mathrm{O}_{2}$ for $30 \mathrm{~min}$, and then incubated with peroxidaseconjugated secondary antibodies: ImmPRESS ${ }^{\mathrm{TM}}$ Reagent Kit peroxidase, Anti-Goat Ig and Universal Anti-Mouse/ Rabbit Ig (Vector Laboratories, Burlingame, CA). DAB (3,3'-Diaminobenzidine)was used for visualizing the peroxidase reaction. The slides were coated with Clarion ${ }^{\mathrm{TM}}$ Mounting Medium (C0487-100 ml, Sigma-Aldrich, Inc, St.Louis, MO).

The IHC slides were analysed by two of the researchers (LK and TK), and the cellular localisation of ADAMs was assessed. ADAM expression in each sample was graded from 0 to $3(0=$ no staining, $1=$ mild staining, $2=$ medium staining, $3=$ strong staining) based on the intensity and extensity of the staining.

\section{Statistical analysis}

Statistical analysis was conducted with SPSS Statistics 22 (SPSS Inc., Chicago, IL). Results are given as mean and standard error of mean (SEM) range, or median and range. Normality of the distributions was assessed with Kolmogorov-Smirnov test. Parametric tests were used for the normally distributed variables, and non-parametric tests otherwise. The unpaired (patients to controls, cell lines) or paired (intrinsic proximal controls) Student's $t$ test was used for normally distributed variables, whereas the MannWhitney $U$ test or the Wilcoxon signed-rank test was used for the non-normal data. To compare the different stages of GERD, ANOVA with Dunnett's test to correct for multiple comparisons were used as parametric tests and the Kruskall-Wallis and Dunn's test (to correct for the multiple comparisons) as non-parametric tests. The JonckheereTerpstra test was used to evaluate ADAM expression during the control-esophagitis-BE-BE with dysplasiaadenocarcinoma sequence. $p$ value $\leq 0.05$ was considered statistically significant, and two-tailed tests were used.

\section{Results}

\section{ADAM mRNA expression in cell lines}

The expression of ADAM9 mRNA was increased in both the Barrett's adenocarcinoma cell line OE33, and the

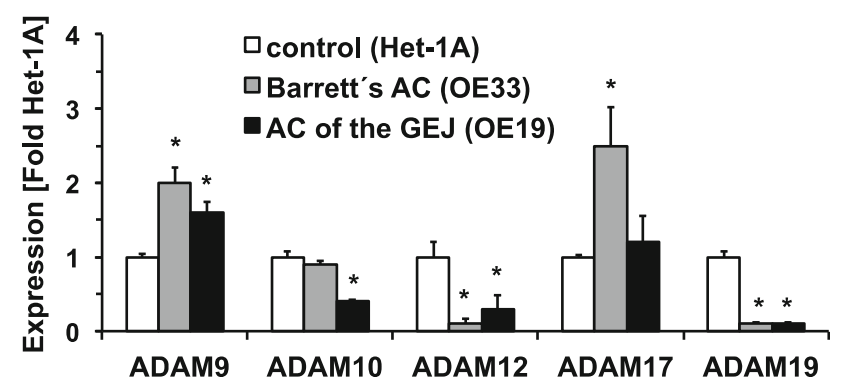

Fig. 1 ADAM mRNA expression in esophageal cell lines relative to expression in Het-1A, and corrected with GAPDH-expression. Stars indicate $t$ test comparisons with significant difference $(p \leq 0.05)$ between the cell line in question, and Het-1A. Error bars represent the standard error of mean. $A C$ adenocarcinoma, GEJ gastroesophageal junction

gastro-esophageal junctional adenocarcinoma cell line OE19 as compared to Het-1A (Fig. 1). For ADAM10, its mRNA expression did not differ in OE33, but decreased in OE19. The mRNA expressions of ADAM12 and ADAM19 were decreased in both OE33 and OE19. The expression of ADAM17 mRNA was increased in OE33, but did not differ in OE19.

\section{ADAM protein expression in cell lines}

The expression of the precursor and mature forms of ADAM proteins was analysed and shown for ADAMs 9 and 10 in Fig. 2.

For ADAM9, two precursor forms (at $110 \mathrm{kD}$ and 100 $\mathrm{kD}$ ), and two mature forms (at $80 \mathrm{kD}$ and $75 \mathrm{kD}$ ) were observed. The expression the mature form at $80 \mathrm{kD}$ was decreased in $\operatorname{OE} 33(p=0.0031)$ and $\operatorname{OE} 19(p=0.0026)$, and that of the mature form at $75 \mathrm{kD}$ was increased in OE33 ( $p<0.001$ ) (Fig. 2a). For ADAM10, the expression of both the precursor form (at $80 \mathrm{kd}$ ) and the mature form (at $60 \mathrm{kD}$ ) were increased in OE33 (precursor form, $p=0.0011$; mature form, $p<0.001$ ) and OE19 (precursor form, $p=0.0095$; mature form, $p<0.001$ ), relative to the squamous control Het-1A (Fig. 2b). For ADAM12, only a precursor form was detected, and no change in its expression was seen (data not shown). For ADAM17, both a precursor form at $110 \mathrm{kD}$ and a mature form ad $90 \mathrm{kD}$ were detected, with no change in their expression, however (data not shown). For ADAM19, only small fragments of the protein were seen (data not shown).

\section{ADAM mRNA expression in patient samples}

The ADAM9 mRNA expression in distal esophagus differed between groups $(p=0.002)$, and was increased in $\mathrm{BE}(p=0.004), \mathrm{BE}$ with dysplasia $(p=0.014)$, and in esophageal adenocarcinoma $(p=0.043)$, relative to 
a

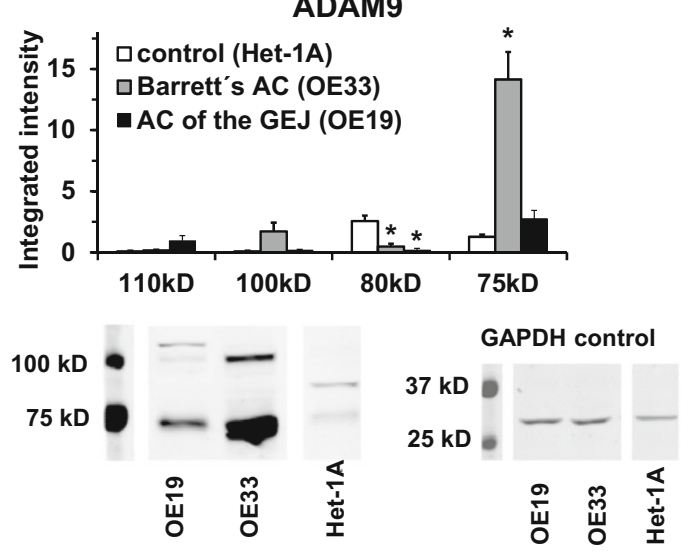

b

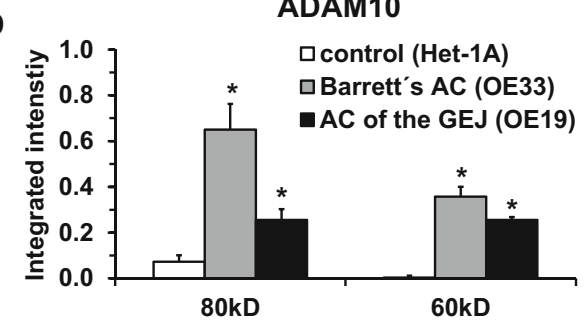

$75 \mathrm{kD}$

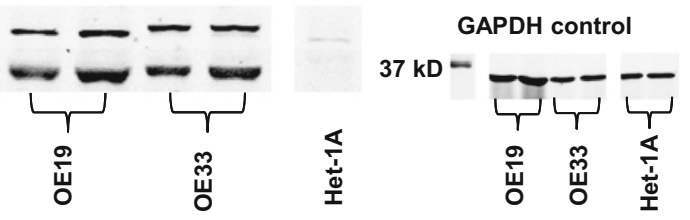

Fig. 2 Protein expression of ADAM9 $\mathbf{a}$ and ADAM10 b in esophageal cell lines by western blotting. A representative western blot, and the corresponding standardisation blot of GAPDH from the same nitrocellulose membrane, are shown below the densitometric results $(N=4-6)$. $T$ test comparisons to Het-1A with significant difference $(p \leq 0.05)$ are indicated with stars. Error bars represent SEM (standard error of mean). $A C$ adenocarcinoma, GEJ gastroesophageal junction

controls (Fig. 3a). The trend test showed a rising trend along the progressive stages of GERD from the control group to $\operatorname{EAC}(p=0.001)$. When compared to proximal intrinsic controls, esophagitis $(p=0.015), \mathrm{BE}(p<0.001)$ and EAC $(p=0.018)$ showed increased ADAM9 expression.

The ADAM10 mRNA expression in distal esophagus varied between groups $(p=0.007)$, but the difference was not statistically significant for any single group (Fig. 3b). There was neither rising nor declining trend in the different stages of GERD. When compared to proximal intrinsic controls, ADAM10 mRNA expression in BE was higher than in these patients' proximal esophagus $(p=0.001)$.

The ADAM12 mRNA expression in distal esophagus differed between groups $(p<0.001)$. It was increased in esophagitis $(p=0.038)$, BE with dysplasia $(p=0.001)$, and EAC ( $p<0.001)$, relative to normal controls (Fig. 3c). In the trend test, there was a rising trend in ADAM12 expression $(p<0.001)$. In comparison to the proximal intrinsic controls, dysplastic BE $(p=0.039)$ and EAC $(p=0.001)$ showed increased ADAM12 expression.

The ADAM17 mRNA expression in distal esophagus, also, differed between groups $(p=0.001)$. It was increased in EAC ( $p=0.004)$ (Fig. 3d). Neither rising nor declining trend during the GERD sequence was noted, and there were no differences relative to the proximal intrinsic controls.

The ADAM19 mRNA expression in distal esophagus differed between groups $(p=0.002)$. ADAM19 mRNA was increased in dysplastic $\mathrm{BE}(p=0.044)$ and in EAC $(p=0.001)$ (Fig. 3e). The trend test showed a rising trend in ADAM19 expression $(p<0.001)$. When compared to proximal intrinsic controls, esophagitis $(p<0.001)$ and non-dysplastic BE $\quad(p=0.025)$ showed increased ADAM19 expression.

\section{Correlation with patient characteristics}

The mRNA expression of ADAMs in the distal esophagus did not differ between the groups with or without neoadjuvant treatment (data not shown). Their expression was also not associated with cancer differentiation (good, moderate, poor) or dysplasia grade (mild, severe) (data no shown). ADAM9 expression was increased in Siewert1 cancers as compared to Siewert2 cancers (shown in Online Resource 2). ADAM12 was increasingly expressed in severe esophagitis as compared to mild esophagitis, as were also ADAM17 and ADAM19 (shown in Online Resource 2).

\section{ADAM protein expression in patient samples}

Normal esophageal squamous epithelium did not show distinct expression of ADAM 9, 10, or 17 proteins. ADAM12 was rarely seen in apically centred cytoplasmic granules. Only ADAM19 was mildly to moderately expressed in the cytoplasm of esophageal squamous cells. At whole, ADAM expression was centred in the glandular cells of the metaplastic/dysplastic esophageal epithelium, and was localized mostly in the cytoplasm but in EAC also occasionally in the nucleus.

ADAM9 was expressed in BE (Fig. 4c) and Barrett's dysplasia, with no increased staining relative to controls (for dysplasia, $p=0.077$, median 1 , range $1-1$ in dysplasia vs. median 0.5 , range $0-1$ in controls) or from $\mathrm{BE}$ to dysplasia. EAC showed increased ADAM9 protein expression relative to controls (median 1 , range $1-2$ in EAC vs. median 0.5 , range $0-1$ in controls, $p=0.026$ ) (Fig. 4a, d). ADAM9 was not differently expressed in esophagitis (Fig. 4b). 

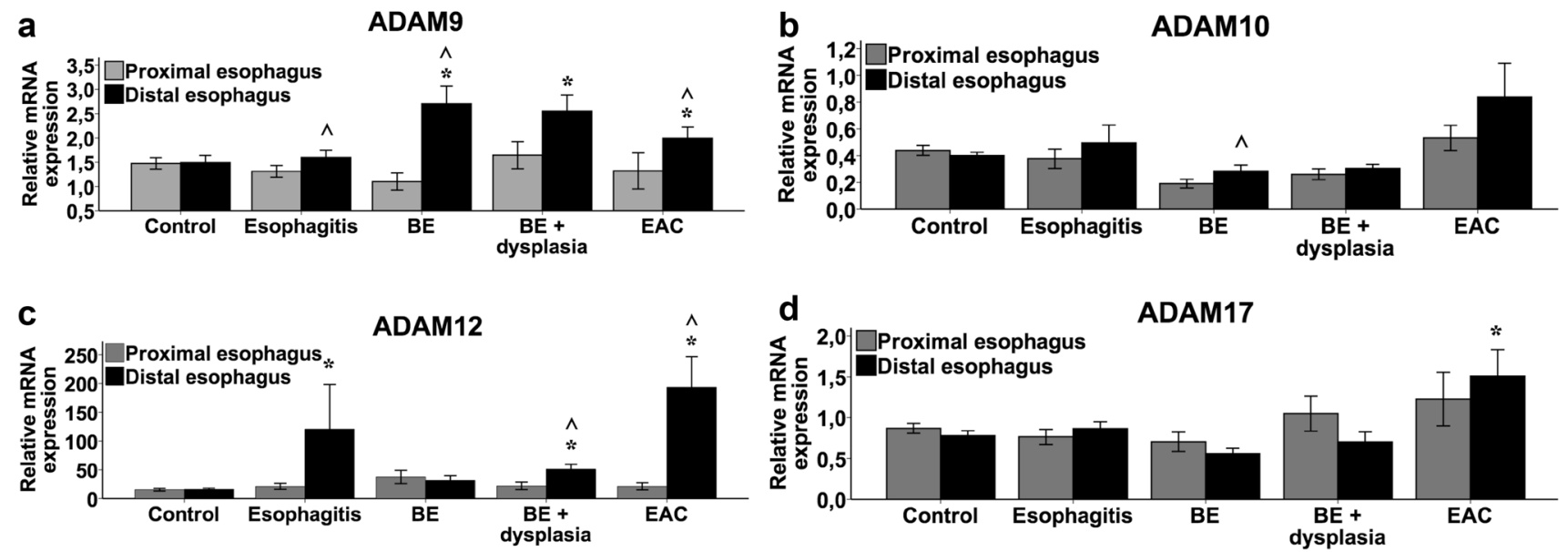

d

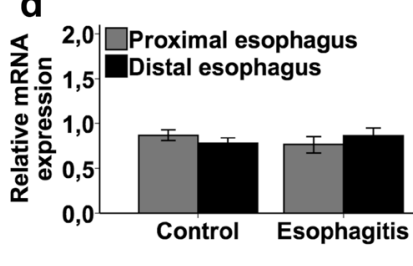

ADAM17

e

ADAM19

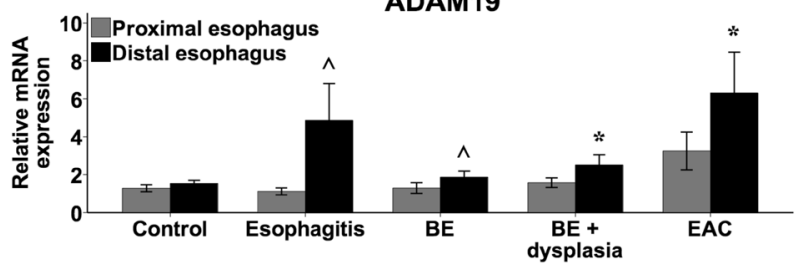

Fig. 3 ADAM mRNA expression in patient samples of different stages of GERD in both the distal and proximal esophagus. Error bars represent the standard error of mean. Stars indicate ANOVA/ Dunnett's or Kruskal-Wallis/Dunn's test comparisons with significant difference $(p \leq 0.05)$ between the patient group and controls, and hat symbol indicates paired $t$ test or Wilcoxon signed rank test comparisons with significant difference $(p \leq 0.05)$ between the distal and proximal samples of the same patient group. Values represent relative expression as compared to the expression in NCI-N87 cell line, corrected for the mean of GAPDH and beta actin expression in the same samples. ADAM9 (a) ADAM10 (b) ADAM12 (c) ADAM17 (d) ADAM19 (e)
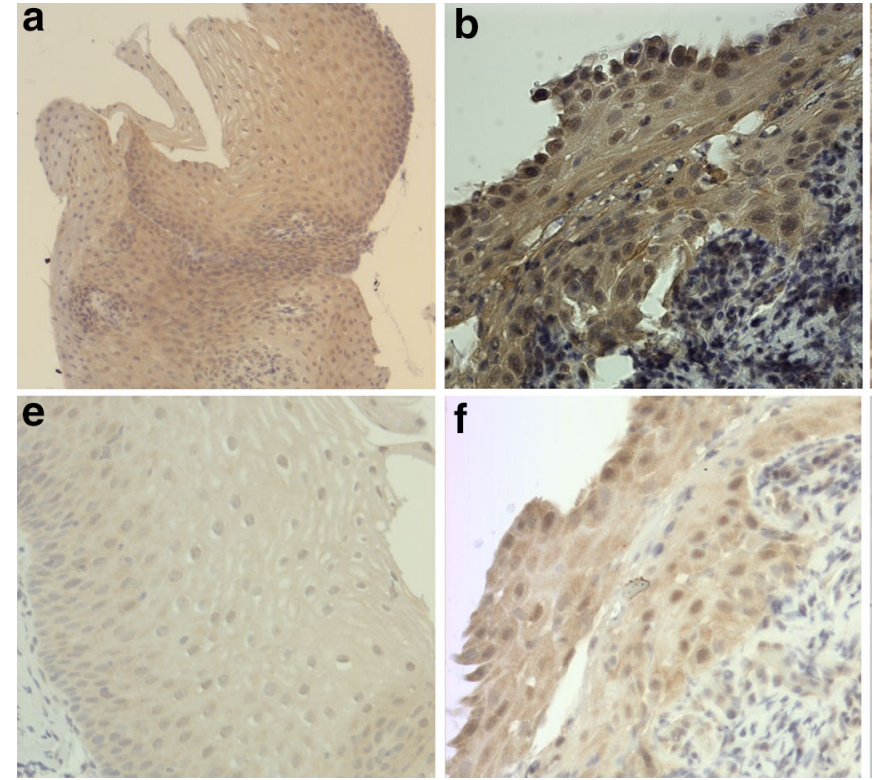

Fig. 4 ADAM9 protein expression in normal squamous mucosa of distal esophagus (original magnification $\times 100)(\mathbf{a})$, in esophagitis $(\times 200)(\mathbf{b})$, in Barrett's esophagus $(\times 100)(\mathbf{c})$, and in esophageal adenocarcinoma $(\times 100) \quad($ d). ADAM10 expression in normal

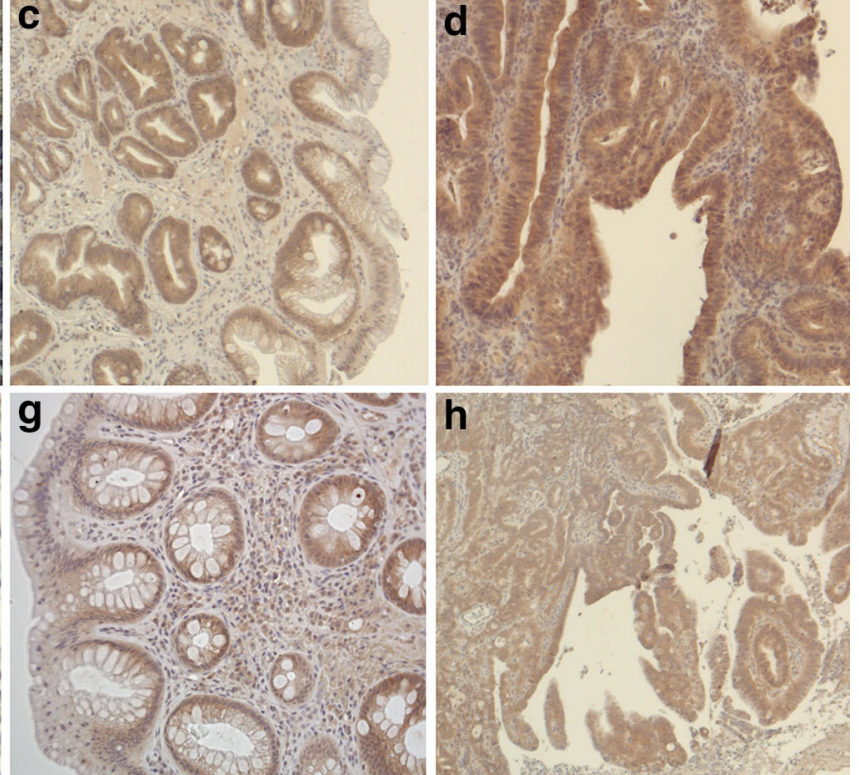

squamous mucosa of distal esophagus $(\times 200)(\mathbf{e})$, in esophagitis $(\times 200)(\mathbf{f})$, in Barrett's esophagus $(\times 100)(\mathbf{g})$ and in esophageal adenocarcinoma $(\times 40)(\mathbf{h})$ 
ADAM10 expression showed a tendency for basal localisation in BE, while in Barrett's dysplasia its expression was more evenly distributed. In EAC, ADAM10 was increasingly expressed relative to controls (Fig. 4e, h) (median 2, range 1-2 in EAC vs. median 1, range 0-1 in controls, $p=0.021$ ), while for dysplasia there was no increase (median 1, range 1-2 in dysplasia vs. median 1, range $0-1$ in controls, $p=0.061$ ), and neither for $\mathrm{BE}$ (Fig. 4g). ADAM10 was not differently expressed in esophagitis (Fig. 4f).

The significant increase of ADAM12 mRNA in severe esophagitis was not reflected at the protein level. Statistically, ADAM12 expression did not differ from normal esophageal squamous epithelium at any stage of GERD.

ADAM17 was increasingly expressed in BE relative to normal controls (median 2, range 2-2 in BE vs. median 0.5, range $0-1$ in controls, $p=0.024$ ), while for Barrett's dysplasia or EAC there was no difference in the expression relative to controls.

ADAM19 was expressed at all stages of GERD (including severe esophagitis), with a tendency for transformation from basally centred staining in BE to apically centred staining in EAC. Statistically, however, the ADAM19 expression in BE/dysplasia/EAC did not differ from the controls.

\section{Discussion}

To our knowledge, this is the first study to examine the ADAM expression in the various phases of GERD preceding cancer, and in EAC also at the protein level. ADAM9 was increased in EAC, and in BE and BE with dysplasia at mRNA level. Other ADAMs studied showed alterations as well, and may participate in the complex network of interactions leading to EAC development.

In EAC, both ADAM9 mRNA and protein expression were elevated. Also in the cancer cell lines, ADAM9 mRNA was elevated, and in the Barrett's adenocarcinoma cell line, ADAM9 protein was elevated. The demonstrated functions of ADAM9 in cancer include promoting cellular adhesion and invasion, as well as interaction with integrins [7]. It can bind to the $\alpha 6 \beta 4$-integrin, the $\alpha 6$-subunit of which is upregulated in EAC [7, 17]. ADAM9 has been found increased e.g. in gastric and prostate cancer, and in pancreatic ductal adenocarcinoma [18-20]. There is preliminary evidence of ADAM9 being involved in invasion/ metastasis [21-23]. Oxidative stress, which is also increased in EAC and its predecessors [24], induces the expression of ADAM9 [20], which also contributes to the cellular invasion induced by oxidative stress [25]. Given the potential contribution of ADAM9 for the cancer metastasising, and the availability of an inhibitor [7], it remains an interesting candidate for further studies assessing its function in GERD and EAC.

ADAM10 protein but not mRNA expression was increased in EAC both in the cell lines and in human samples. This might be caused by some posttranscriptional or posttranslational modulation leading to e.g. increased translation of mRNA, or decreased degradation of the protein. As shown before for ADAM17, posttranscriptional mechanisms may alter ADAM protein expression without a change in mRNA level by altering the protein half-life [26]. Previously, however, an increase in ADAM10 mRNA has been found in esophago-gastric carcinomas [14]. ADAM10 expression has been linked to many human cancers [7]. Possible mechanisms for promoting carcinogenesis in EAC include its ability to cleave tumour necrosis factor alpha (TNF- $\alpha$ ) [27], increased along the metaplasia-dysplasia-carcinoma-sequence [28], and E-cadherin [29], leading to increased free $\beta$-catenin levels, and thereby increased expression of the proto-oncogene C-myc, also found upregulated in Barrett's adenocarcinoma $[28,30]$. It might also act by cleaving the adhesion molecule CD44 [7], also shown to be associated with EAC [17].

In human samples, ADAM12 mRNA was increased in esophagitis, BE with dysplasia, and in EAC. ADAM19 showed a similar expression profile, except for that in esophagitis and $\mathrm{BE}$ it was increased only relative to intrinsic controls. These elevations were not, however, seen at the protein level. It is possible that in these tissues the prevailing form of these ADAMs was not captured by our IHC protocol. ADAM12 may exist as a membrane-anchored long form, or as a soluble secreted form [31]. As ADAM12 and ADAM19 mRNA were highly expressed in esophagitis, their increased expression in EAC might reflect the inflammatory changes seen, and possibly of importance for the development of a malignancy, such as EAC [32, 33]. Our results are in accordance with the previous study of esophago-gastric adenocarcinomas [14]. ADAM12 and ADAM19 expression have been linked to both inflammatory diseases and cancer [7, 11, 31, 34, 35].

ADAM17 mRNA, but not protein, was increased in EAC in human samples, and in the Barrett's adenocarcinoma cell line OE-33. IHC may not have captured the relatively small increase in mRNA. Tumours also showed marked heterogeneity in the expression of ADAM17 protein. Previously, ADAM17 mRNA has been found increased in esophago-gastric adenocarcinomas [14]. This molecule is one of the most studied ADAM-molecules and is upregulated in many human cancers [7]. It cleaves TNF$\alpha$, and also TGF- $\beta$, both cytokines of possible importance in $\mathrm{EAC}[7,13,36]$.

We compared the ADAM expression in GERD and EAC to both normal controls and to the proximal unaffected 
mucosa of the patients. The previous study of esophagogastric adenocarcinomas compared the ADAM expression in cancer to "peri-tumoural normal tissue" [14]. We used statistical correction for the multiple comparisons problem, which may have affected our results in comparison to studies without these corrective methods. Our results were, however, mostly in accordance with the previous study showing increased ADAM mRNA expression in esophagogastric adenocarcinomas [14].

A limitation of our study was the relatively small number of samples, especially in the preliminary characterisation of ADAM expression at the tissue level by IHC. The elevated ADAM mRNA levels were not always seen at the protein level. This may be at least partly due to ADAMs being subjects to significant post-transcriptional and post-translational regulation [8]. This kind of modulation, leading possibly to altered levels of specific isoforms, altered function, or altered localisation, may even be an important regulatory mechanism controlling the cellular and inter-cellular actions of ADAMs.

The major disparities between ADAM expression in the cell lines studied and the human samples are not surprising, given the individual nature of molecule expression in each cancer and heterogeneity across each tumour [37]. In human samples, a factor likely to affect the molecular expression is the stroma and its interactions with the cancer cells [38]. With the small number of samples, neoadjuvant treatment did not affect ADAM expression. Our patients were a little older than the controls, and there was a male predominance in patients in contrast to the male proportion of only $35 \%$ in the controls. This is likely to mostly reflect the age and gender as known risk factors of BE and EAC [39]. To our knowledge, no reports of age- or gender-dependent differences in ADAM expression in the adult have been reported, but a possible bias cannot be excluded.

We have shown that the expression of this multifunctional ADAM protein group is altered in EAC and during its development. ADAMs are linked to inflammatory processes and to many molecules already shown to be dysregulated along the development of EAC. Even a minor modification in the expression of regulatory molecules, such as ADAMs, may have a significant impact. Future studies with more samples, data on protein levels, association with prognostic factors and fate, and functional assays, are needed to define the role of ADAMs in esophageal carcinogenesis.

Acknowledgments The authors wish to thank doctors Eero Sihvo, Juha Kauppi, Tarja Peräkylä and Perttu Arkkila for their invaluable assistance in providing part of the patient samples in the General Thoracic and Esophageal Surgery clinic, Heart and Lung Centre, Helsinki University Hospital, and in the Department of Gastroenterology, Helsinki University Hospital. The authors also wish to thank
Mrs. Yvonne Sundström for her skilful technical and secretarial assistance. The authors did not receive any writing assistance.

Funding The study was financially supported by grants from the Sigrid Jusélius Foundation (no grant number) and Helsinki University Hospital Research Funds (TYH 2012131 and TYH 2014240).

\section{Compliance with ethical standards}

Conflict of interest The authors declare that they have no conflict of interest.

Informed consent Informed consent was obtained from all individual participants included in the study.

Ethical approval All procedures performed in studies involving human participants were in accordance with the ethical standards of the institutional and/or national research committee and with the 1964 Helsinki declaration and its later amendments or comparable ethical standards.

\section{References}

1. Holmes RS, Vaughan TL. Epidemiology and pathogenesis of esophageal cancer. Semin Radiat Oncol. 2007;17:2-9.

2. Parrilla P, Martinez de Haro LF, Ortiz A, Munitiz V, Molina J, Bermejo J, et al. Long-term results of a randomized prospective study comparing medical and surgical treatment of Barrett's esophagus. Ann Surg. 2003;237:291-8.

3. Spechler SJ, Lee E, Ahnen D, Goyal RK, Hirano I, Ramirez F, et al. Long-term outcome of medical and surgical therapies for gastroesophageal reflux disease: follow-up of a randomized controlled trial. JAMA. 2001;285:2331-8.

4. Corey KE, Schmitz SM, Shaheen NJ. Does a surgical antireflux procedure decrease the incidence of esophageal adenocarcinoma in Barrett's esophagus? A meta-analysis. Am J Gastroenterol. 2003;98:2390-4.

5. Prasad GA, Bansal A, Sharma P, Wang KK. Predictors of progression in Barrett's esophagus: current knowledge and future directions. Am J Gastroenterol. 2010;105:1490-502.

6. Garton KJ, Gough PJ, Raines EW. Emerging roles for ectodomain shedding in the regulation of inflammatory responses. J Leukoc Biol. 2006;79:1105-16.

7. Mochizuki S, Okada Y. ADAMs in cancer cell proliferation and progression. Cancer Sci. 2007;98:621-8.

8. Murphy G. The ADAMs: signalling scissors in the tumour microenvironment. Nat Rev Cancer. 2008;8:929-41.

9. Duffy MJ, Mullooly M, O’Donovan N, Sukor S, Crown J, Pierce A, et al. The ADAMs family of proteases: new biomarkers and therapeutic targets for cancer? Clin Proteomics. 2011;8:9.

10. Duffy MJ, McKiernan E, O'Donovan N, McGowan PM. Role of ADAMs in cancer formation and progression. Clin Cancer Res. 2009;15:1140-4.

11. Franze E, Caruso R, Stolfi C, Sarra M, Cupi ML, Ascolani M, et al. High expression of the "A Disintegrin And Metalloprotease" 19 (ADAM19), a sheddase for TNF-alpha in the mucosa of patients with inflammatory bowel diseases. Inflamm Bowel Dis. 2013;19:501-11.

12. Yoshimura T, Tomita T, Dixon MF, Axon AT, Robinson PA, Crabtree JE. ADAMs (a disintegrin and metalloproteinase) messenger RNA expression in Helicobacter pylori-infected, normal, and neoplastic gastric mucosa. J Infect Dis. 2002; 185:332-40.

13. Lagarde SM, ten Kate FJ, Richel DJ, Offerhaus GJ, van Lanschot JJ. Molecular prognostic factors in adenocarcinoma of the esophagus and gastroesophageal junction. Ann Surg Oncol. 2007;14:977-91.

14. Baren JP, Stewart GD, Stokes A, Gray K, Pennington CJ, O'Neill R, et al. mRNA profiling of the cancer degradome in oesophago-gastric adenocarcinoma. Br J Cancer. 2012;107:143-9.

15. Rokkas T, Pistiolas D, Sechopoulos P, Robotis I, Margantinis G. Relationship between Helicobacter pylori infection and esophageal neoplasia: a meta-analysis. Clin Gastroenterol Hepatol. 2007;5:1413-7, 7 e1-2.

16. Boonstra JJ, van Marion R, Beer DG, Lin L, Chaves P, Ribeiro C, et al. Verification and unmasking of widely used human esophageal adenocarcinoma cell lines. J Natl Cancer Inst. 2010;102:271-4.

17. Nair KS, Naidoo R, Chetty R. Expression of cell adhesion molecules in oesophageal carcinoma and its prognostic value. J Clin Pathol. 2005;58:343-51. 
18. Carl-McGrath S, Lendeckel U, Ebert M, Roessner A, Rocken C. The disintegrin-metalloproteinases ADAM9, ADAM12, and ADAM15 are upregulated in gastric cancer. Int J Oncol. 2005;26:17-24.

19. Grutzmann R, Luttges J, Sipos B, Ammerpohl O, Dobrowolski F, Alldinger I, et al. ADAM9 expression in pancreatic cancer is associated with tumour type and is a prognostic factor in ductal adenocarcinoma. $\mathrm{Br} \mathrm{J}$ Cancer. 2004;90:1053-8.

20. Sung SY, Kubo H, Shigemura K, Arnold RS, Logani S, Wang R, et al. Oxidative stress induces ADAM9 protein expression in human prostate cancer cells. Cancer Res. 2006;66:9519-26.

21. Mazzocca A, Coppari R, De Franco R, Cho JY, Libermann TA, Pinzani M, et al. A secreted form of ADAM9 promotes carcinoma invasion through tumorstromal interactions. Cancer Res. 2005;65:4728-38.

22. Shintani Y, Higashiyama S, Ohta M, Hirabayashi H, Yamamoto S, Yoshimasu $\mathrm{T}$, et al. Overexpression of ADAM9 in non-small cell lung cancer correlates with brain metastasis. Cancer Res. 2004;64:4190-6.

23. Xu Q, Liu X, Cai Y, Yu Y, Chen W. RNAi-mediated ADAM9 gene silencing inhibits metastasis of adenoid cystic carcinoma cells. Tumour Biol. 2010;31:217-24.

24. Sihvo EI, Salminen JT, Rantanen TK, Ramo OJ, Ahotupa M, Farkkila M, et al. Oxidative stress has a role in malignant transformation in Barrett's oesophagus. Int J Cancer. 2002;102:551-5.

25. Mongaret C, Alexandre J, Thomas-Schoemann A, Bermudez E, Chereau C, Nicco C, et al. Tumor invasion induced by oxidative stress is dependent on membrane ADAM 9 protein and its secreted form. Int $\mathbf{J}$ Cancer. 2011;129:791-8

26. Santiago-Josefat B, Esselens C, Bech-Serra JJ, Arribas J. Post-transcriptional up-regulation of ADAM17 upon epidermal growth factor receptor activation and in breast tumors. J Biol Chem. 2007;282:8325-31.

27. Hikita A, Tanaka N, Yamane S, Ikeda Y, Furukawa H, Tohma S, et al. Involvement of a disintegrin and metalloproteinase 10 and 17 in shedding of tumor necrosis factor-alpha. Biochem Cell Biol. 2009;87:581-93.

28. Tselepis C, Perry I, Dawson C, Hardy R, Darnton SJ, McConkey C, et al Tumour necrosis factor-alpha in Barrett's oesophagus: a potential novel mechanism of action. Oncogene. 2002;21:6071-81.
29. Reiss K, Ludwig A, Saftig P. Breaking up the tie: disintegrin-like metalloproteinases as regulators of cell migration in inflammation and invasion. Pharmacol Ther. 2006;111:985-1006.

30. Tselepis C, Morris CD, Wakelin D, Hardy R, Perry I, Luong QT, et al. Upregulation of the oncogene c-myc in Barrett's adenocarcinoma: induction of c-myc by acidified bile acid in vitro. Gut. 2003;52:174-80.

31. Taniguchi T, Asano Y, Akamata K, Aozasa N, Noda S, Takahashi T, et al. Serum levels of ADAM12-S: possible association with the initiation and progression of dermal fibrosis and interstitial lung disease in patients with systemic sclerosis. J Eur Acad Dermatol Venereol. 2013;27:747-53.

32. Saadi A, Shannon NB, Lao-Sirieix P, O'Donovan M, Walker E, Clemons NJ, et al. Stromal genes discriminate preinvasive from invasive disease, predict outcome, and highlight inflammatory pathways in digestive cancers. Proc Natl Acad Sci USA. 2010;107:2177-82.

33. Kavanagh ME, O'Sullivan KE, O'Hanlon C, O'Sullivan JN, Lysaght J, Reynolds JV. The esophagitis to adenocarcinoma sequence; the role of inflammation. Cancer Lett. 2014;345:182-9.

34. Kerna I, Kisand K, Suutre S, Murde M, Tamm A, Kumm J, et al. The ADAM12 is upregulated in synovitis and postinflammatory fibrosis of the synovial membrane in patients with early radiographic osteoarthritis. Joint Bone Spine. 2014;81:51-6.

35. Rocks N, Paulissen G, El Hour M, Quesada F, Crahay C, Gueders M, et al Emerging roles of ADAM and ADAMTS metalloproteinases in cancer. Biochimie. 2008;90:369-79.

36. Mendelson J, Song S, Li Y, Maru DM, Mishra B, Davila M, et al. Dysfunctional transforming growth factor-beta signaling with constitutively active Notch signaling in Barrett's esophageal adenocarcinoma. Cancer. 2011;117:3691-702.

37. Gerlinger M, Rowan AJ, Horswell S, Larkin J, Endesfelder D, Gronroos E, et al. Intratumor heterogeneity and branched evolution revealed by multiregion sequencing. N Engl J Med. 2012;366:883-92.

38. Pietras K, Ostman A. Hallmarks of cancer: interactions with the tumor stroma. Exp Cell Res. 2010;316:1324-31.

39. Spechler SJ, Souza RF. Barrett's esophagus. N Engl J Med. 2014;371:836-45. 